\title{
A new architecture for advanced telematics services
}

\author{
A. Piccolo \& V. Galdi \\ Dipartimento di Ingegneria dell'Informazione ed Ingegneria Elettrica, \\ Università degli Studi di Salerno, Italy
}

\begin{abstract}
Advances in Information and Communication Technologies have helped spread the use of telematics, together with a fall in price and a rise in reliability of the systems. The application of such technologies to the mobility area can support the growing requirements for efficiency and safety of transportation systems by means of the development of new services and the strengthening of the existing ones, improving the effectiveness of transportation systems.

Therefore, for a meaningful diffusion of telematics in transportation, a new approach to the Intelligent Transportation Systems (ITS) design and implementation is required, moving away from the pioneering era where each project is developed like a prototype, towards a new one characterized by a wide use of international standards able to guarantee interoperability and expandability amongst the existing systems.

Much work has been done and a lot remains in progress for the definition of a shared standard for the ITS architecture.

This paper outlines ARTIST, the Italian ITS architecture, and proposes a physical architecture of the next generation for telematics services, investigating its potential application in the info-mobility area.

The proposed physical layer for infomobility services is based on a fully decentralized architecture, able to work without a control centre and characterized by high computational power and a very effective communication and multimedia features.
\end{abstract}

All the devices are based on standard technologies, whereas the communication system is based on Wi-Fi standard, exclusively.

The proposed architecture has been applied and tested in SITUS-TP, a research project for the public transportation pointed out by the authors at the Campus of the University of Salerno.

Keywords: ITS - Intelligent Transport Systems, telematics, information systems, Transport Telematics Architecture, XML. 


\section{Introduction}

The growth of the mobility demand characterizes more and more our present time: in Europe over the past 25 years goods traffic has increased by $75 \%$ and passenger movements by $110 \%$ [1]. This implies problems with energy consumption, traffic and air pollution, with heavy impacts on the quality of life, and it calls for a new model of mobility able to guarantee a sustainable growth.

Modal redressing, low environmental impact vehicles and infrastructures strengthening seem to be the most important choices to solve today's mobility problems. Therefore, if the building of new infrastructures does not seem a feasible solution, especially in the medium and short-term due to the high environmental impact and the very high financial resources involved, a more efficient approach should be represented by the Intelligent Transportation Systems (ITS) [1-3].

The ITS have become more and more popular in recent decades with the development of telecommunication, information and computer technologies, offering an alternative to the traditional approaches for addressing transportation problems and for complying with user needs.

The ITS include the technologies to supply advanced telematics services for a better use of existing mobility resources: in particular, ITS offer a great variety of services such as automated traffic management (ATM), traveler information and trip planning services, automatic vehicle control and monitoring (AVC and AVM), incident and emergency management, etc., with the advantage of $[4-6]$ :

- a drop in road casualties;

- a travel time saving;

- a more efficient inter-modal transport chains;

- a growth in road safety;

- a smaller ecological impact.

Nevertheless, the design and the implementation of these systems are not trivial due to the difficulty in integrating system parts that must work together to accomplish the same task $[7,8]$. Moreover, today, many telematics systems for transportation are implemented like autonomous projects, carried out independently. This approach causes problems both in terms of the inter-system integration, due to the lack of interoperability between the equipments and data, and in terms of upgradeability, due to the lack of standards adopted in design and in implementation phases.

This suggests the use of frameworks and of common standards to encode services and functions, data flows, communication protocols and physical interfaces, like an ITS Architecture.

A lot of architectures have been implemented in the world, like the NITSA in USA, Karen in the UE, ACTIF in France, ARTIST in Italy, and others, each of them tailored to specific needs connected to different geography, demographic and institutional arrangements.

In this context, ARTIST, the Italian ITS Architecture, plays a significant role. ARTIST is an initiative of the Italian Ministry of Transport and Infrastructure 
created with the aim to help and harmonize the development of the local market, in order to achieve the integration of transport modes and ITS systems, both at national and European level.

In this paper an application of ARTIST to the infomobility area and an innovative physical architecture for ITS is proposed. In detail, in section 2 an overview of the main ITS architectures is given, whereas a description of the Italian Telematics Architecture is proposed in section 3. In section 4 an application of ARTIST to SITUS-TP is presented, an infomobility research project carried out in the campus of Fisciano of the University of Salerno by the authors. The project is based on an innovative fully decentralized architecture, able to work without a control centre and characterized by high computational power, and by very effective communication and multimedia features.

\section{ITS architectures}

An ITS architecture provides a common structure for the design of telematic systems for intelligent transportation systems. It defines the framework that describes how system components interact and work together to achieve the total system goals and around which many different design approaches can be developed, each one tailored to meet specific needs [9].

Moreover, system architecture defines the functions that must be performed by components or subsystems, where these functions work and what information is exchanged among the components.

There are many benefits to be gained in the transportation community through application of an ITS architecture, that:

- ensures interoperability between components and consistency of information delivered to end-users;

- permits an appropriate level of technology independence and it allows new technologies to be incorporated easily;

- correlates needs and problems to services that must be performed, providing traceability in the project of all the user needs and the aspirations of the customers;

- highlights efficiencies by eliminating redundancy of functions and data flows and it simplifies the identification of services and functionalities that may not have been initially considered to satisfy the aspirations of the customer.

In order to achieve this purpose, an ITS architecture provides a systematic mechanism for capturing the objectives and requirements of all parties involved, such as public authorities, transport operators and final users [10].

The main elements of an ITS architecture are the User Needs, consisting of a wide collection of requirements of users, operators, travelers and providers, involved in ITS deployment; such requirements are usually referred to as ITS Stakeholders,.

A Conceptual Model shows the whole system and explains how it works; at this level the system can be viewed as a single entity connected to the outside world through Terminators: each Terminator defines the functionality to be 
provided by the outside world and the "data" to receive from, or to be provided by, the external world.

The definition of the system functionalities is contained in the Functional or Logical Architecture. This architecture performs the system as a series of functions and data stores that must fulfill the User Needs and must interface with the functionalities provided by or to the Terminators. The functions communicate among them, with data stores and with Terminators through functional data flows modeled in Data Flow Diagrams (DFD). They are used to show how the data flows link the functions, the data stores and the terminators.

The functions in the Logical Architecture are grouped together to form physical entities in the Physical Architecture.

The Physical Architecture provides a framework for deploying and operating all the user needs and offers a framework to describe how to interconnect subsystems and interfaces, ensuring interoperability. All physical entities are linked together and to the Terminators by physical data flows. A Communication Architecture defines the communication behaviors of the data links amongst the physical subsystems of the Physical Architecture.

Following these guidelines, with the spread of ITS, more and more National or regional ITS Architectures have been defined.

The first National ITS Architecture was pointed out in the United States in the late 1996. The American National ITS Architecture (NITSA) provides a common framework for planning, defining and integrating intelligent transportation systems for a period at least of twenty years $[3,11]$.

The NITSA is today a mature product that reflects the contributions of a broad cross-section of the ITS community (transportation practitioners, systems engineers, system developers, technology specialists, consultants, etc.). It is considered an architecture deployment-oriented and market or equipment oriented system, instead of service oriented. This approach still remains a peculiarity of the American architecture.

The guidelines of the European ITS Framework Architecture were pointed out in the KAREN project, founded by the European Commission and updated and extended by the FRAME project $[12,13]$.

The European ITS Framework Architecture is designed to provide a flexible high-level framework that individual countries can tailor to their own requirements and user needs. The main objective of FRAME is to stimulate and co-ordinate the intelligent transportation systems in Europe in wide-scale deployment. One way used to meet these challenges is to require all stakeholders to adhere to an agreed European Framework in order to accommodate national plans and to support the various efforts in research, standardization, deployment and investment. Furthermore, FRAME furnishes the guiding lines through which a new architecture can be developed at different levels of detail (national, regional, city area, etc.) [16].

National ITS Architecture projects based on the European ITS Framework Architecture are ACTIF (Architecture Cadre pour le Transports Intelligents en France) in France [14, 15], TTS-A in Austria, TEAM (Telematics Eurodigibus And Mobility) in Czech Republic and ARTIST in Italy [16]. 


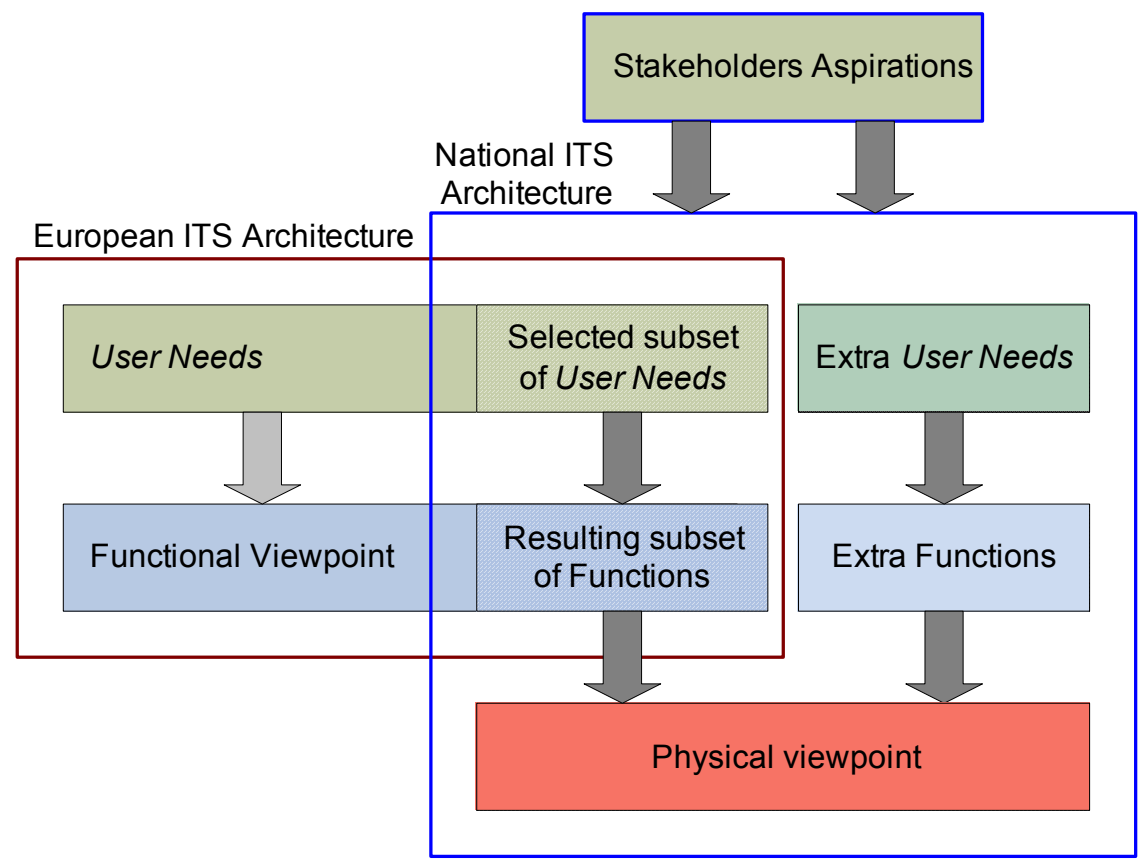

Figure 1: European vs. National ITS architectures.

As said before, ARTIST is an initiative of Italian Ministry of Transport and Infrastructure, and its aim is to help and harmonize the development of the local market, in order to achieve the integration of transport modes and ITS services, both at National and European level.

\section{ARTIST - the Italian ITS architecture}

The ARTIST project comes from the National Plan for Transport and Logistics (PGTL) [2] and from the European project FRAME with the aim to promote the development of efficient and interoperable transportation system in Italy.

ARTIST's main objective is to provide public authorities, transport operators, infrastructure managers and private companies with general guidelines to simplify and to improve in effectiveness and efficiency the design, the development, the management and the maintenance of ITS systems.

The first version of ARTIST was released in March 2003 and it represents the result of a structured development process where the most important National parties active in the ITS have been involved in. Such involvement has given to ARTIST a further added-value by making it a shared reference framework which has been consolidated and validated by institutions at National level.

ARTIST describes all components of an ITS system, how they interact and work together and what information they exchange. On the bases of User Needs, ARTIST defines: 
- the required services for users;

- the functional, logical and physical relationships among systems;

- information flows exchanged among systems;

- organizational relationships among the different users involved in the development and management of ITS.

The main innovative elements of ARTIST are:

- the extensions to multimodality/intermodality aspects, especially concerning the User Requirements and the Functional Architecture;

- the Organizational Architecture, based on a methodology for clarifying the roles, responsibilities of actors providing the services described by the functions in the Logical Architecture;

- a Navigation Tool, based on an open source approach, in order to make the Architecture usable.

These features can bring many benefits: ARTIST, in fact, is an open architecture and so it allows the easy integration of new functions and updating of the existing ones conferring, in this way, modularity and expansibility to the IT systems.

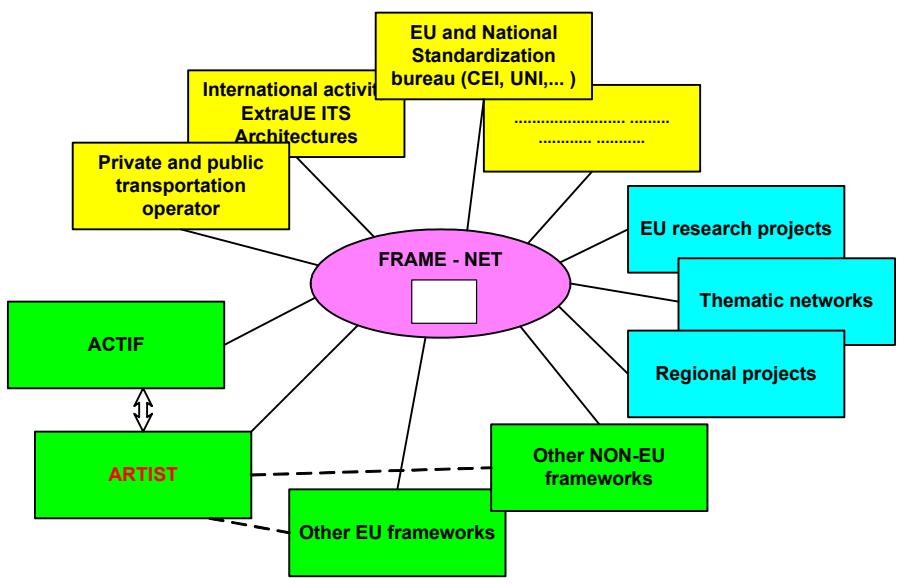

Figure 2: $\quad$ ARTIST and European Architecture.

Through ARTIST it is possible to point out efficient devices and services, aimed at answering user needs, promoting quality and competitiveness of the transportation system. It favors the integration of ITS systems and services, allowing the final user to get each moment updated and reliable information on traffic conditions and transport services. In this way, traveler choices can be more efficient, so promoting safety, transport comfort and pollution reduction.

After all, ARTIST is a European reference element, allowing system producers to get a clear description of the rules to be followed in order to trade competitive systems, both at National and European level. In fact, ARTIST is compatible and compliant with FRAME, the European Reference Architecture, and it's strictly related to the French National Architecture, ACTIF. ARTIST and 
ACTIF adopt the same language and they represent an important step towards the integration of ITS systems at European level, and a model to be followed in other National projects.

ARTIST consists of five main elements:

- As is, that consists of a wide overview of National and International state of the art on ITS;

- User Needs, that represent the requirements of the users of the transportation system which the Architecture must satisfy;

- Logical Architecture, that contains all functionalities and data flows necessary for providing the services identified by the User Needs;

- Physical Architecture, that suggests how the functionalities of the Logical Architecture can be grouped in different physical locations, in order to create a system which can be successfully implemented;

- Organizational Architecture, which stresses the organizational and business aspects which enable the services defined by Logical Architecture.



Figure 3: $\quad$ ARTIST structure.

On the bases of this organization, ARTIST has proposed to carry out an ITS project considering an operative paradigm structured in four steps:

1. to define the User Needs on the bases of the necessary services for the users;

2. to select the Logical Functions and Data Flows, necessary for providing the services defined;

3. to group the Logical Functionalities in different physical locations and to aggregate logical Data Flows making physical Data Flows;

4. to analyze and to define the most relevant organizational, legal and business aspects.

The great usability and the aforementioned advantage connected to the use of the architecture have brought about a growing interest both in the technical and scientific Italian communities and a second release of the architecture is now in progress. 


\section{SITUS-TP project: an application of ARTIST architecture to advanced telematics services}

Notwithstanding the fact that ARTIST is a young architecture, today many ITS applications are developing in Italy according to its guidelines [17]. Out of all the ARTIST applications, SITUS-TP represents one of the most interesting one, both for the innovative physical architecture proposed and for the technologies adopted.


Trasporto Pubblico) is a Telematics Information System of the University of Salerno (UoS) for the Public Transportation developed by the authors at the Department of Electrical Engineering and Computer Science of the University of Salerno according to the ARTIST guidelines.

SITUS-TP tries to solve the following critical states, highlighted by the users of the shuttle service during the first year of duty:

- difficulties perceived to access to the service;

- poor safety perceived by the users at the bus stop late in the evening;

- lack of reliable information.

These critical states have been expressed in user needs selected among those suggested by ARTIST.

On the bases of the identified User Needs and Logical Functions, the Logical Architecture and the Data Flows have been selected by ARTIST and pointed out. In particular, the Functional Areas picked out are:

- Area 4: Manage Public Transport Operation, for the TP services planning and the management of linked information (arrivals, departures, locations, occupations of the buses);

- Area 6: Provide Traveller Journey Assistance, for the process to collect, store and deliver the traveller information (traffic conditions, parking information, route information for the different modes of transport, interesting places on the way).

These areas provide functions able to support the following services for the TP users of the SITUS-TP:

- forecasting and scheduled time of arrival available both at bus stops and on the web;

- information about the current position of the buses related to the bus stop locations available both on the web and at bus stop;

- information at bus stops about didactics, examination date, special events organized by the closest Faculties;

- news, weather reports and other general information at bus stop.

The obtained logical architecture of the SITUS-TP systems is depicted in figure 9 , where it is possible to identify the functions depicted by boxes with smoothed corners, the databases, depicted by the traditional cylinder, the data flows, depicted by arrows, and the terminators, depicted by rectangular boxes.

It is worth noting that the modularity and the expandability of ARTIST have allowed an increase in the architecture, adding on new functionalities related to 
the collection, management and transmission of information and data not strictly connected to the Transportation System. The lack of functions for infomobility services based on extra-system data, like the information not strictly related to the mobility, has suggested extending the ATRIST architecture pointing out a new infomobility service and a new terminator, highlighted in red in figure 10 and shown in detail in figure 4. This has been possible because ARTIST is an "open" architecture that offers the possibility of extending, adding on and improving its functionalities by the integration of "compatible" components and sub-systems.



Figure 4: New infomobility service coded in ARTIST.
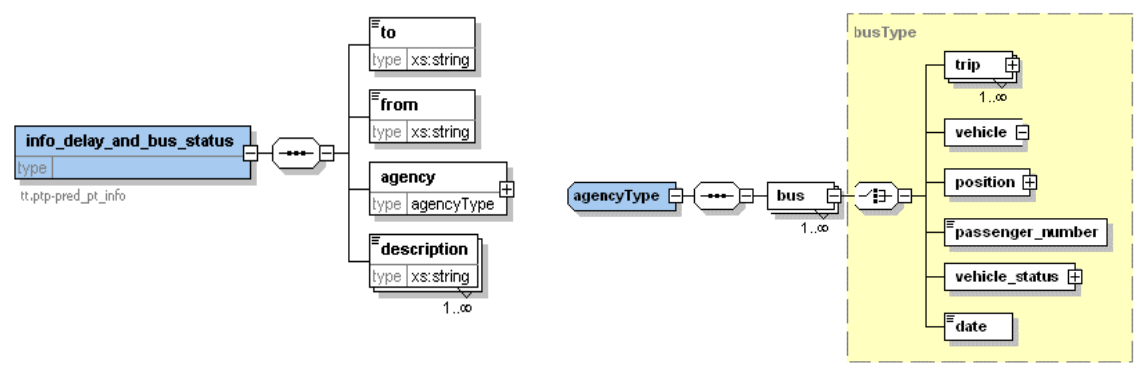

Figure 5: XML coding of Logical data flow.

In the detail, in the new service, coded in compliance with the ARTIST formalism, a new terminator, the "GENERIC INFORMATION SUPPLIER" furnishes the information through the fesp-news_data data flux to the function "Content Management". A further function, the "Content Publishing" selects the information to publish and furnishes them to the "TRAVELLER".

Starting from the logical architecture, the physical layer of SITUS-TP has been pointed out. Unlike the traditional approach, as those described in [18] and depicted in figure 6, the SITUS-TP physical architecture is based on a fully distributed structure that has no need of a control centre, shown in figure 10.

All the devices are based on standard and commercial technologies: a network of Informative Terminals, placed in the bus shelters, provides at the bus stops information about forecasting arrivals at current bus stop, programmed events and news available at the UoS, whereas a web server furnishes both on the web and at the bus stops, forecasting arrivals, bus locations and information about the Academic activities, using an high level interface based on a "virtual reality" representation of the Campus, as shown in figure 7 and 8. 


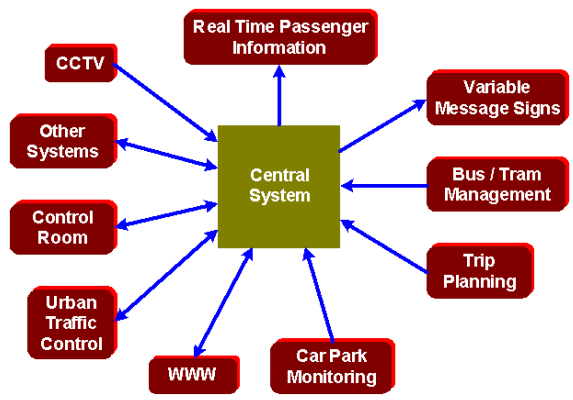

Figure 6: Conventional ITS architecture.


Figure 7: $\quad$ Bus stop info-points.

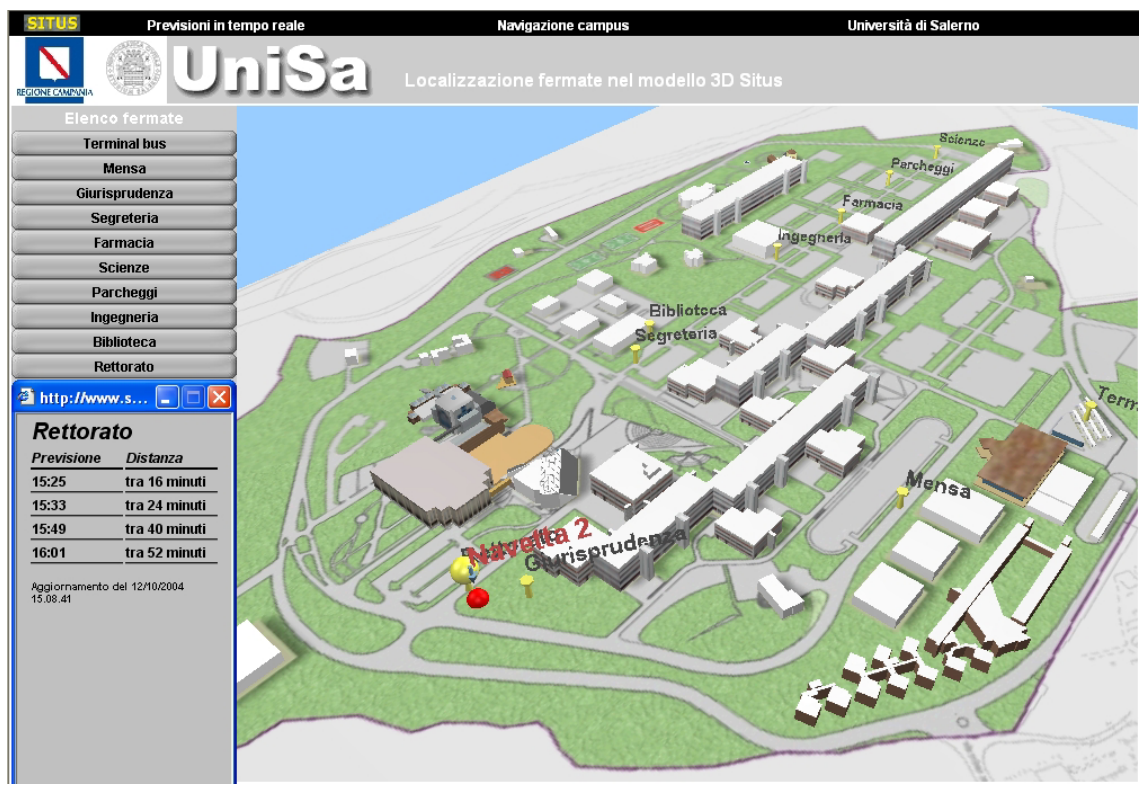

Figure 8: $\quad$ Intranet monitoring and forecasting service.

WIT Transactions on The Built Environment, Vol 89, (C) 2006 WIT Press

www.witpress.com, ISSN 1743-3509 (on-line) 


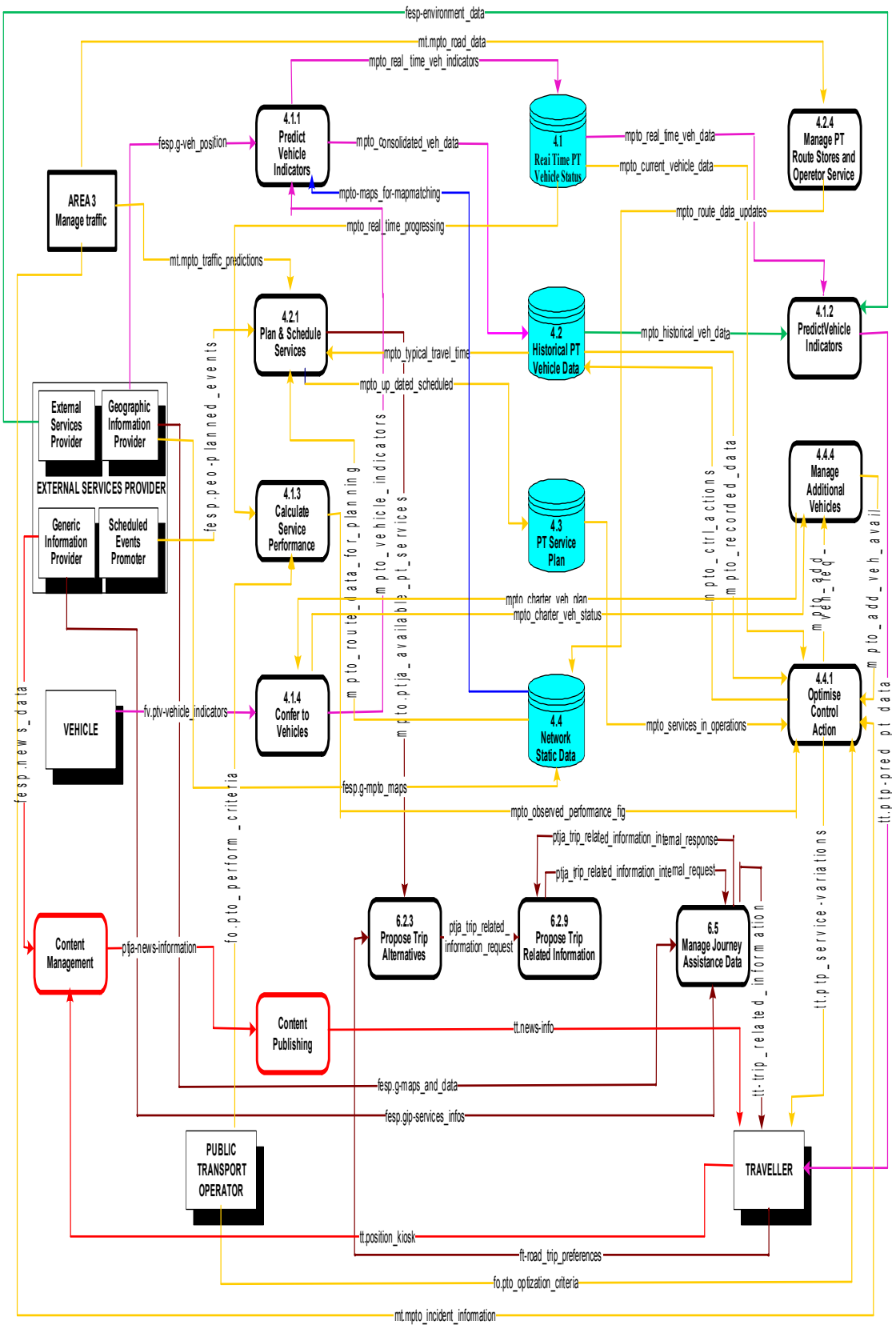

Figure 9: $\quad$ SITUS-TP logical architecture. 


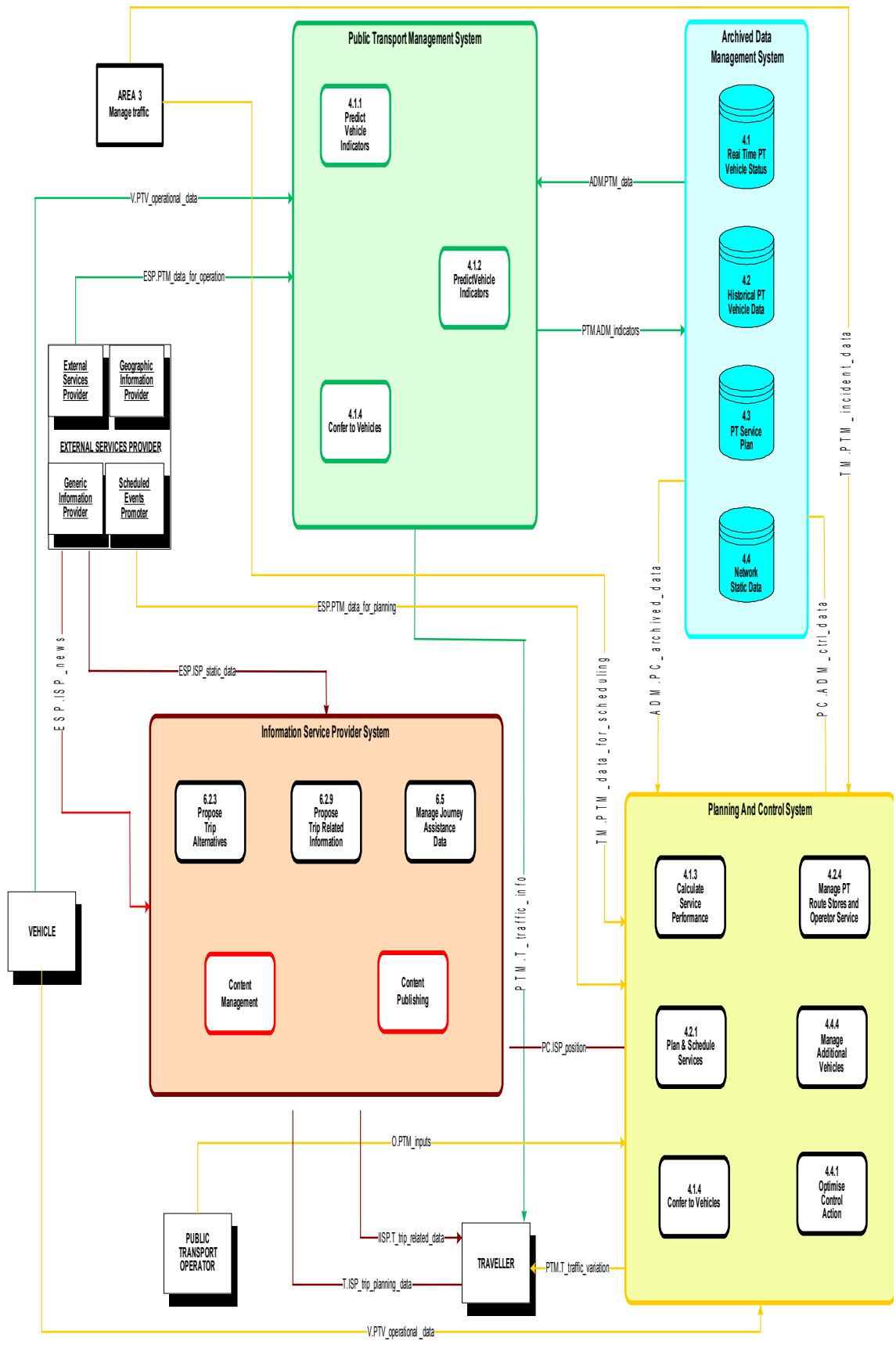

Figure 10: SITUS-TP: physical architecture. 
The communication system is an "all Wi-Fi" architecture that doesn't produce traffic costs. In particular, the communication architecture is a virtual local network based on the Wi-Fi IEEE 802.11g standard, exclusively.

The protocols used for the communication amongst all the intelligent devices of the systems are TCP-IP and UDP.

\section{Conclusions}

The advances in Information and Communication Technologies (ICT) have helped bring about the spread of telematics, with a consequential fall in price and rise in reliability of the related devices and have promoted the support and the growing requirements in efficiency and safety of transportation systems through the development of new telematics services.

To maximize the positiveness related to the spreading of Intelligent Transportation Systems a new approach to the designing and to the implementation of such system is necessary. In the last decade more efforts have been done for the definition of shared standards both for the logical and physical architectures, in order to guarantee interoperability, modularity and scalability of the telematics systems.

The paper has provided the current state of the art of ITS architecture, analyzing, in particular, ARTIST, the recently publisher Italian ITS architecture, investigating on the potential application of ITS in the info-mobility area, especially referred to public transportation systems.

ARTIST has been used to design and implement an innovative infomobility system for public transportation, SITUS-TP, pointed out by the authors at the Campus of the University of Salerno.

SITUS-TP is based on a decentralized architecture, able to work without connection to the control centre and characterized by a high computational power, communication and multimedia features. The project defines an open platform based on standard and low cost telematics technologies and based on the Wi-Fi IEEE $802.11 \mathrm{~g}$ standard for the communication infrastructure exclusively.

\section{Acknowledgements}

The project has been realized with the financial support of the Centro Regionale di Competenza Trasporti of "Regione Campania", of the Assessorato alla Università e Ricerca - Regione Campania and of the Assessorato alle Attività Produttive - Regione Campania.

The authors acknowledge the CSTP, the Salerno's Mobility Company, and the Mizar Automazione S.p.A. for the support furnished in the implementation phase.

\section{References}

[1] UE Commission, White Book - The European Transportation policy up to 2010: the time of the choices, 2001, ISBN 92-894-0343-8. 
[2] Programming and Planning Office of Italian Ministry of Infrastructure and Transportations, Piano Generale dei Trasporti e della Logistica, October 2000.

[3] Architecture Development Team: National ITS Architecture - ITS Vision Statement; Federal Highway Administration US Department of Transportation Washington.

[4] L. Figueredo, I. Jesus, J.A. Tenreiro Machado, J. R. Ferriera, J.L.M. de Carvalho: Towards the Development of Intelligent Transportation Systems.

[5] P. Blythe, T. Rackliff, R. Holland, J. Mageean, The potential for ITS in improving and integrating services at urban transport interchanges for public transport, Proc. of Tenth International Conference on Road Transport Information and Control, 2000, 4-6 April 2000, page 192 - 199.

[6] V. Calderaro, V. Galdi, A. Piccolo: La gestione telematica del Trasporto Pubblico, published in Italian on the Journal AEIT, ISSN 0013-6131, July-August 2004, page 55-61.

[7] A. Piccolo, V. Galdi, A. Vitale, C. Masturzo, Lo standard XML per la condivisione e lo scambio dei dati in applicazioni ITS, published in Italian on the Journal AEIT, ISSN 0013-6131, December 2005.

[8] L.V. Williams, Industry efforts to standardize the car bus, Proc. of ICCE International Conference on Consumer Electronics, 2002, 18-20 June 2002, page $32-33$.

[9] European Commission, Planning a modern transportation system - A guide to intelligent transportation system architecture - IST, Frame project.

[10] L. van den Berg: Standardized Infrastructure for Transportation, University of Valencia, Proc. of International ITS congress, Madrid, 1720 November 2003.

[11] U.S. Department of Transportation Intelligent Transportation Systems Joint: Program Office Developing Intelligent Transportation Systems Using the National ITS Architecture.

[12] KAREN, available at url: http://www.cordis.lu/telematics/tap_transport/ research/projectsum/karen.html

[13] FRAME-NET, available at url: http://www.frame-online.net/home.htm

[14] ACTIF, available at url: http://www.its-actif.org/

[15] H. Mongeot, R. Pagny, Case study: developing an ITS System architecture for France, IEE.

[16] Programming and Planning Office of Italian Ministry of Infrastructure and Transportations, ARTIST - Italian Architecture for ITS, available at url: www.its-artist.rupa.it.

[17] Piccolo, V. Galdi, Applicazione dell'architettura ARTIST per lo sviluppo di sistemi di informativa all'utenza del TPL, published in Italian on the Journal AEIT, ISSN 0013-6131, July/August 2005.

[18] FRAME-NET, About ITS architecture, available at url: http://www.frameonline.net/aboutArchitecture.htm 